\title{
HIV/aids em mulheres jovens em uma regional de saúde do Paraná, Brasil
}

\section{HIV/aids in young women in a health regional in Paraná, Brazil}

Marlise Lima Brandão , Dayeny Fernandes Farago ${ }^{2}$, Maria Marta Nolasco Chaves ${ }^{3}$, Flaviane Marizete Limas ${ }^{4}$, Daiane Siqueira de Luccas ${ }^{5}$, Daiana Kloh Khalaf ${ }^{6}$, Rafaela Gessner Lourenço ${ }^{7}$

1. ORCID: https://orcid.org/0000-0002-2367-2390. Enfermeira. Mestre em Enfermagem. Universidade Federal do Paraná. Programa de Pós-graduação em Enfermagem. Curitiba, Paraná, Brasil.

E-mail: mlblise@gmail.com

2. ORCID: https://orcid.org/0000-0002-5921-9571. Enfermeira. Universidade Federal do Paraná. Programa de Residência Integrada Multiprofissional em Atenção Hospitalar - Área de concentração Saúde da Mulher. Curitiba, Paraná, Brasil.

E-mail: dayenyfarago@gmail.com

3. ORCID: https://orcid.org/0000-0001-8952-5695. Enfermeira. Doutora em Enfermagem. Universidade Federal do Paraná. Programa de Pós-graduação em Enfermagem. Curitiba, Paraná, Brasil.

E-mail: mnolascochaves@gmail.com

4. ORCID: https://orcid.org/0000-0002-2718-5143. Enfermeira. Mestre em Enfermagem. Universidade Federal do Paraná. Programa de Pós-graduação em Enfermagem. Curitiba, Paraná, Brasil.

E-mail: flavianemlimas@gmail.com

5. ORCID: https://orcid.org/0000-0002-9173-8128. Enfermeira. Doutora em Enfermagem. Universidade Federal do Paraná. Programa de Pós-Graduação em Enfermagem. Curitiba, Paraná, Brasil.

E-mail: enfdailuccas@gmail.com

6. ORCID: https://orcid.org/0000-0001-5770-7523. Enfermeira. Doutora em Enfermagem. Universidade Federal do Paraná. Departamento de Enfermagem. Curitiba, Paraná, Brasil.

E-mail: daianakloh@gmail.com

7. ORCID: https://orcid.org/0000-0002-3855-0003. Enfermeira. Doutora em Ciências. Universidade Federal do Paraná. Departamento de Enfermagem. Curitiba, Paraná, Brasil.

E-mail: rglufpr@gmail.com

CONTATO: Autor correspondente: Marlise Lima Brandão | Endereço: Av.

Prefeito Lothário Meissner, 632, Campus Botânico, Bloco Didático II, 3ํandar Jardim Botânico - Curitiba /PR | Telefone: (41)98801-2990

E-mail: mlblise@gmail.com 


\section{RESUMO}

As características da epidemia de HIV/aids denotam sua determinação histórica e social. O objetivo desse estudo foi descrever as características da epidemia de HIV/aids em mulheres adultas jovens, moradoras de municípios da $2^{\text {a }}$ Regional de Saúde do Paraná. Trata-se de um estudo ecológico retrospectivo, com coleta de dados em setembro de 2017, no Sinan NET. Amostragem intencional, que incluiu mulheres de 20 a 29 anos, moradoras da 2 ${ }^{\mathrm{a}}$ Regional de Saúde do Paraná, notificadas com HIV/aids de 2007 a 2015. A análise ocorreu à luz da Determinação Social do Processo Saúde-doença. Foram 602 casos de HIV, 24 de aids e 22 de óbitos em mulheres de 20 a 29 anos; dentre as 648 notificadas, $72,5 \%$ eram da raça/cor branca, $18,2 \%$ com ensino médio, $21,2 \%$ eram donas de casa e $32,6 \%$, gestantes. O desafio para o enfrentamento do agravo é trabalhar na sua determinação social, ou seja, na escolaridade, renda, ocupação e relações de gênero.

DESCRITORES: Epidemia. HIV/aids. Mulher. Saúde Coletiva. Enfermagem.

\section{ABSTRACT}

The characteristics of the HIV/AIDS epidemic denote its historical and social determination. This study described the characteristics of the HIV/AIDS epidemic in young adult women living in municipalities of the 2nd Health Regional Office of Paraná. This is a retrospective ecological study, with data collection in September 2017, in Sinan NET. Intentional sampling, which included women aged 20 to 29 years, residents of the 2nd Health Regional of Paraná, notified with HIV/AIDS from 2007 to 2015. The analysis occurred considering the Social Determination of the Health-Disease Process. There were 602 cases of HIV, 24 of AIDS and 22 of deaths in women aged 20 to 29 years; among 648 notified, $72.5 \%$ were white, $18.2 \%$ with high school education, $21.2 \%$ were housewives and $32.6 \%$ were pregnant. The challenge for facing the grievance is to work on its social determination, i.e., on schooling, income, occupation, and gender relations.

DESCRIPTORS: Epidemic. HIV/aids. Women. Collective Health. Nursing. 


\section{INTRODUÇÃO}

$\Delta$

infecção pelo Vírus da Imunodeficiência Humana (HIV) é um fenômeno de

magnitude internacional. Mudanças nas características da epidemia denotam sua determinação histórica e social, associadas, sobretudo, às desigualdades sociais, o que permite verificar a associação entre gênero, raça e classe social para a conformação de um cenário de vulnerabilidade social para aqueles que o integram ${ }^{1-3}$.

O Programa Conjunto das Nações Unidas sobre HIV/aids (Unaids) tem registrado que, até 2020, aproximadamente 36,7 milhões de pessoas viviam com HIV no mundo; as novas infecções representando cerca de 1,5 milhão, das quais $50 \%$ foram em mulheres ${ }^{2}$. No Brasil, até junho de 2020, foi registrado aproximadamente 1 milhão de casos pessoas vivendo com HIV/aids no país ${ }^{3}$.

No início da epidemia de aids, em 2009, a razão entre os sexos (M:F) era de 15 homens para cada dez mulheres e, em 2019, último dado epidemiológico, eram 3,7 homens para cada mulher com a síndrome, na faixa etária de 20 a 29 anos $^{3}$.

Refletir sobre a determinação do processo de feminilização do HIV/aids no contexto histórico, social, cultural e econômico do Brasil é necessário para possibilitar o desenvolvimento de ações de prevenção e assistência que possibilitem o enfrentamento do fenômeno ${ }^{3}$. Mulheres que vivem em contextos sociais desiguais, por exemplo, as que habitam bolsões de pobreza nos centros urbanos - que concentram os maiores índices de analfabetismos, as exclusões étnicas, a comercialização e o uso de drogas ilícitas - têm, no seu cotidiano, processos que potencializam as vulnerabilidades para as infecções sexualmente transmissíveis (ISTs) $)^{1,4}$.

Neste estudo, adotou-se o conceito de vulnerabilidade proposto por Nakamura et al. (5:120), que é "entendida como uma dimensão tanto dos processos de exclusão, discriminação ou enfraquecimento dos grupos sociais, quanto da capacidade de enfrentamento dessas condições".

No período de 2007 a 2015, no Brasil, foram registrados 46.764 casos de HIV e 134.684 casos de aids em mulheres. Neste grupo, 59,6\% das mulheres autodeclaram-se da raça/cor parda ou preta e para $96,4 \%$ a forma de contaminação foi a relação sexual heterossexual. Chama a atenção que, no ano de 2015, a razão de sexos foi de 2,4 homens para cada mulher com HIV/aids, dado que reafirma a feminilização da epidemia ${ }^{3}$. Especificamente para a faixa etária de 20 a 29 anos, o 
total de mulheres vivendo com HIV no Brasil era de 14.164 e daquelas vivendo com aids era de 28.989, no período de 2007 a $2015^{3}$. Cabe destacar que os dados apresentados são do mesmo período de dados utilizados nesta pesquisa, para contextualizar a realidade desse momento histórico da epidemia de HIV e aids.

Em relação ao número de notificações em gestantes vivendo com HIV, no mesmo período, foi de 61.584 casos, com a seguinte distribuição: $40,0 \%$ se declararam da raça/cor branca, 53,2\% tinham entre 20-29 anos e 26,9\% apresentaram ensino fundamental incompleto. A taxa de detecção em gestantes no ano de 2015 foi de 2,7 casos/mil nascidos vivos ${ }^{3}$.

Convergente com os dados nacionais, o Paraná, passa por um processo de feminilização da epidemia de HIV/aids. No estado, a razão dos casos no ano de 1986 era de 15/1; em 1996 foi de 3/1 e, em 2014, chegou a 2,4 homens para cada mulher com HIV/aids ${ }^{3,6}$.

A Secretaria do Estado de Saúde do Paraná notificou, entre os anos de 2007 e 2015, 1.655 casos de gestantes vivendo com HIV. Esses foram em maior número na Segunda Regional de Saúde (2 $2^{\mathrm{a}}$ RS), onde $70,7 \%$ dos casos eram de gestantes vivendo com HIV, seguida pela $9^{\mathrm{a}}$ e $3^{\mathrm{a}}$ Regionais de Saúde, correspondendo cada uma a $10,5 \%$ dos casos, e, depois, a $10^{\text {a }}$ RS, que correspondeu a $8,7 \%$ casos de gestantes vivendo com HIV?.

Em relação a casos de transmissão vertical, a taxa de detecção no estado caiu de 2,32 casos/100 mil hab. em 2007 para 0,69 casos/100 mil hab. no ano de 2015, uma redução de $70 \%{ }^{7}$.

Na perspectiva da saúde coletiva, considerada campo de práticas e saberes, e, ainda, área de conhecimento que se constitui como espaço privilegiado para discutir práticas e ações que promovam a vida e a saúde de indivíduos e coletivos, com vistas à prevenção de agravos, a saúde e a doença são compreendidas como processo e não como estados estanques de uma mesma realidade ${ }^{5,8}$.

A determinação do processo saúde é compreendida a partir de processos históricos e sociais que ocorrem nas dimensões que conformam a realidade, a partir da inserção do homem na produção e das relações que daí decorrem e conformam a sua reprodução social8-9. 
Com base no exposto e entendendo a necessidade de a enfermagem refletir sobre a determinação social e propor intervenções ${ }^{5}$, o presente estudo tem como objetivo descrever as características da epidemia de HIV/aids em mulheres adultas jovens, moradoras de municípios da Segunda Regional de Saúde do Paraná.

\section{MÉTODO}

Trata-se de um estudo epidemiológico do tipo ecológico retrospectivo ${ }^{10}$. A coleta de dados ocorreu com dados secundários do Sistema de Informação de Agravos de Notificação versão para Internet (Sinan-Net). Foram variáveis de interesse: sexo, idade, município de moradia, raça/cor, escolaridade, zona de moradia, ocupação, provável modo de transmissão, evolução do caso. A amostragem foi do tipo intencional, com coleta de dados no mês de setembro de 2017. Como critérios de inclusão para o estudo consideraram-se as notificações de mulheres vivendo com HIV/aids (MVHAs), moradoras de municípios da $2^{\text {a }}$ RS do Paraná, na faixa etária de 20 a 29 anos, no período de 2007 a 2015 . Foram excluídas todas as notificações em duplicidade.

A $2^{\text {a }}$ RS do Paraná é composta pelos 29 municípios da região metropolitana de Curitiba, são eles: Adrianópolis, Agudos do Sul, Almirante Tamandaré, Araucária, Balsa Nova, Bocaiúva do Sul, Campina Grande do Sul, Campo do Tenente, Campo Largo, Campo Magro, Cerro Azul, Colombo, Contenda, Curitiba, Doutor Ulysses, Fazenda Rio Grande, Itaperuçu, Lapa, Mandirituba, Piên, Pinhais, Piraquara, Quatro Barras, Quitandinha, Rio Branco Do Sul, Rio Negro, São José dos Pinhais, Tijucas do Sul e Tunas do Paraná7.

Essa RS abriga 30,9\% da população paranaense, com 2.956 .269 (91,7\%) pessoas em zona urbana e 267.567 (8,3\%) em zona rural. É a regional que detém os municípios com maior e menor Índice de Desenvolvimento Humano Municipal (IDHM) do Paraná, Curitiba e Doutor Ulysses, respectivamente ${ }^{7}$.

Os dados coletados foram organizados por meio do programa Microsoft Exce ${ }^{\odot}$ 2016 e tabulados em planilha dinâmica, a fim de tratar, agrupar e comparar os elementos, para, assim, proceder à análise descritiva das informações à luz da Determinação Social do Processo Saúde-Doença ${ }^{8-9}$. 
A categorização da ocupação utilizou a Classificação Brasileira de Ocupações (CBO) do Ministério do Trabalho e Emprego ${ }^{11}$.

A pesquisa em tela faz parte do projeto intitulado: "A epidemia de HIV/aids em adultos jovens em uma Regional de Saúde do Paraná, sob a ótica da Epidemiologia Crítica". Foram seguidos os parâmetros da Resolução no. 466/2012 do Conselho Nacional de Saúde ${ }^{12}$ e a pesquisa foi aprovada pelo Comitê de Ética em Pesquisa do Setor de Ciências da Saúde da Universidade Federal do Paraná, sob o Parecer de oㅡ 1.988.988. A coparticipação da Secretaria de Estado da Saúde do Paraná foi aprovada pelo Comitê de Ética em Pesquisa do Hospital do Trabalhador/SESA, sob o Parecer $n^{\circ}$ 2.035.675. O estudo foi liberado do preenchimento do Termo de Consentimento Livre e Esclarecido, pois não foram coletadas informações que possibilitassem a identificação do indivíduo notificado.

\section{RESULTADOS}

No período de 2007 a 2015, o Paraná apresentou um total de 4.061 notificações de HIV/aids em indivíduos entre 20 e 29; desses casos, 1.144 (28,2\%) são de pessoas do sexo feminino, na $2^{\mathrm{a}} \mathrm{RS}, 2.512(61,9 \%)$ pessoas que viviam com HIV/aids, na faixa etária do estudo.

Os dados mostraram que, dos 1.144 casos em mulheres que viviam com HIV/aids no estado, 648 casos (56,6\%) estavam concentrados na $2^{\text {a }}$ RS. Entre as 648 MVHAs moradoras da $2^{\mathrm{a}} \mathrm{RS}, 615$ (94,9\%) eram casos de HIV, 29 (4,5\%), de aids e 4 $(0,6 \%)$ eram registros de óbitos com causa HIV/aids.

Tabela 1. Número de casos de mulheres vivendo com HIV/aids na $2^{\mathrm{a}}$ Regional de Saúde, segundo o ano de notificação.

\begin{tabular}{lllll}
\hline Ano & HIV & Aids & Óbitos & $\%$ \\
\hline 2007 & 16 & 0 & 0 & 2,5 \\
2008 & 41 & 1 & 1 & 6,6 \\
2009 & 35 & 1 & 0 & 5,6 \\
2010 & 31 & 4 & 2 & 5,7
\end{tabular}




$\begin{array}{lllll}2011 & 25 & 2 & 1 & 4,3 \\ 2012 & 31 & 2 & 2 & 5,4 \\ 2013 & 59 & 1 & 6 & 10,2 \\ 2014 & 110 & 3 & 4 & 18,0 \\ 2015 & 254 & 10 & 6 & 41,7 \\ \text { Total } & \mathbf{6 0 2} & \mathbf{2 4} & \mathbf{2 2} & \mathbf{6 4 8}(\mathbf{1 0 0 \% )}\end{array}$

Elaborado pelas autoras (2019).

Os cinco municípios que concentraram o maior número de casos em mulheres foram: Curitiba com 358 (55,2\%) casos, Colombo com 48 (7,4\%), Pinhais com 40 (6,2\%), São José dos Pinhais com 35 (5,4\%) e Piraquara com 33 (5,1\%) casos. Destaca-se que os Municípios de Tijucas do Sul e Agudos do Sul não tiveram notificações de MVHAs no período.

Os dados apontaram que, das 648 MVHAs analisadas, 361 (55,7\%) tinham entre 25-29 anos de idade e 470 (72,5\%) se declararam de raça/cor branca. Quanto ao nível de escolaridade, $118(18,2 \%)$ apresentaram ensino médio completo e 117 $(18,0 \%)$, o ensino fundamental incompleto.

Da amostra deste estudo, teve-se que 68 (10,5\%) foram classificadas como trabalhadoras dos serviços, vendedoras do comércio em lojas e mercados (Grupo 5), seguindo-se ocupações em serviços administrativos (Grupo 4) com 15 (2,3\%) notificações e $33(5,1 \%)$ foram classificadas nos grupos $1,2,3,6,7,8$ e $9^{11}$.

$E m$ relação às ocupações que não são classificadas pela $C B O$, mas são codificadas para inserção no SINAN-Net, as MVHAs classificadas como donas de casa totalizaram $138(21,3 \%)$ casos, as que estavam desempregadas somaram 24 $(3,7 \%)$, e $15 \quad(2,3 \%)$ casos foram codificados como estudantes e presidiárias/confinadas. Todavia, destaca-se que 355 (54,8\%) das Fichas de Notificações Individuais (FNIs) tiveram o campo ocupação ignorado ou não preenchido.

Quanto ao provável modo de transmissão, 584 (90,1\%) notificações indicaram a contaminação por relação sexual com homens, seguidas de $25(3,9 \%)$ por uso de 
drogas injetáveis, seis $(0,9 \%)$ por transmissão vertical, seis $(0,9 \%)$ por relação sexual com mulheres, cinco $(0,8 \%)$ por transfusão sanguínea, quatro $(0,6 \%)$ por relação sexual com homens e mulheres, e em 18 (2,8\%) notificações o campo provável modo de transmissão foi ignorado.

Outro dado que se destacou no estudo foi que, das 648 MVHAs notificadas, $211(32,6 \%)$ eram gestantes e, destas, $144(22,2 \%)$ tiveram o diagnóstico tardio, a partir do segundo trimestre de Idade Gestacional (IG). Entre as 211 gestantes, 210 $(99,5 \%)$ viviam com HIV e uma $(0,5 \%)$, com aids, sendo essa última residente no Município de Curitiba. Os cinco municípios que mais notificaram os casos em gestantes foram: Curitiba com 124 (58,8\%) casos, Almirante Tamandaré com 15 (7,1\%), Colombo com 14 (6,6\%), Fazenda Rio Grande com 11 (5,2\%), e Piraquara com $10(4,7 \%)$ casos.

\section{DISCUSSÃo}

A $2^{\text {a }}$ RS reúne $62 \%$ dos casos de HIV/aids na população de $20-29$ anos do Estado do Paraná. As notificações de MVHAs concentram-se nas residentes de Curitiba, que é o município com maior concentração populacional da regional e do estado, com $358(55,2 \%)$ casos, semelhante às regiões metropolitanas de Porto Alegre e São Paulo, que agregam, respectivamente, $56,9 \%$ e 54,0\% do total de casos de seus estados ${ }^{13-14}$.

Quando observado o número de notificações por ano, verifica-se que os anos de 2013, 2014 e 2015 foram os que concentraram maior número de casos de MVHAs na $2^{\mathrm{a}} \mathrm{RS}$, divergindo da realidade nacional, na qual esses mesmos três anos perfazem $11,3 \%, 12,5 \%$ e 12,8\% das notificações em MVHAs de 20-29 anos, no período de 2007 a $2015^{3}$. Esse dado permite inferir que, no Paraná, há uma sensibilidade maior para o diagnóstico nesses últimos três anos do estudo, fenômeno que pode estar relacionado à implantação da notificação dos casos de HIV em 2014.

Em relação à raça declarada nas notificações, diverge da estatística nacional, na qual $59,6 \%$ das mulheres se declararam pretas e pardas ${ }^{3}$. Porém, o dado analisado coincide com a realidade da população local, uma vez que a região metropolitana de 
Curitiba apresenta $74,5 \%$ da sua população geral autodeclarados da raça/cor branca, condição associada à colonização da região, que é de origem europeia ${ }^{15}$.

No que concerne aos dados sobre a escolaridade das MVHAs deste estudo, a realidade observada na pesquisa é diferente da encontrada no estudo realizado em um município do Maranhão ${ }^{16}$, no qual $40 \%$ das notificações por HIV/aids eram em indivíduos com ensino fundamental incompleto e 10,5\% tinham ensino médio completo, uma realidade que retrata as diferenças nas regiões brasileiras.

Embora a Portaria MS o 3.947 de 25 novembro de $1998^{17}$ estipule que a ocupação na $\mathrm{FNI}$ deva ser preenchida de acordo com a $\mathrm{CBO}^{11}$, os Boletins Epidemiológicos que apresentam dados sobre o fenômeno HIV/aids não contemplam discussões acerca desta informação, o que impossibilita a realização de comparações com os achados do estudo em tela, ${ }^{3,6,13-14,18}$. Pontua-se que as ocupações com as maiores frequências nas notificações analisadas convergem com a escolaridade encontrada na amostra, tendo em vista que a escolaridade mais frequente foi ensino médio completo.

Esses resultados convergem com estudo acerca do processo de feminização do HIV/aids no Brasil e que indica a relação de maior vulnerabilidade das mulheres para as doenças sexualmente transmissíveis devido à desigualdade social e à discriminação de gênero. Portanto, é necessário discutir as relações de poder e gênero, que prejudicam a vida das mulheres, de forma a investir no empoderamento feminino, valioso para transformação social e, mudança dos padrões hegemônicos de dominação das mulheres pelos homens ${ }^{1}$.

Sobre o provável modo de transmissão do vírus, 90\% das MVHAs declararam, na notificação, que a infecção ocorreu por meio da relação sexual heterossexual. Estes dados são convergentes com a realidade nacional, na qual 96,4\% das MVHAs declararam essa mesma categoria de exposição ${ }^{3}$. Ademais, esses dados apontam para outro tema relevante no âmbito da saúde, o início da prática sexual sem proteção entre as mulheres ${ }^{19}$.

Neste estudo foram identificados 211 casos de gestantes, dessas, $68,2 \%$ tiveram diagnóstico tardio, o que caracteriza um problema para a eliminação nacional da transmissão vertical do HIV, conforme problematizado por estudo que abordou, entre outros aspectos, a magnitude da transmissão vertical do HIV no Estado do Rio Grande do Sul20. 
O Ministério da Saúde referenda a captação da gestante no primeiro trimestre de gestação para vinculação no pré-natal, momento de solicitação de exames para apoio diagnóstico, dentre os quais está o teste anti-HIV. Uma vez diagnosticada como portadora do vírus, a recomendação é iniciar o tratamento antirretroviral para suprimir a carga viral e, assim, diminuir a possibilidade da transmissão vertical. Porém, a despeito da captação e do tratamento precoce, não se pode desconsiderar a possibilidade de contaminação tardia com o HIV, o que aumenta a vulnerabilidade tanto para as gestantes, como de seus futuros filhos ${ }^{21}$.

Apesar de a maioria dos casos das gestantes que integraram este estudo ter sido notificada a partir do segundo trimestre da gestação, considera-se que a assistência integral à mulher deva ocorrer também fora do período gravídicopuerperal. Portanto, há que se refletir sobre orientações educativas que estimulem os indivíduos a buscarem apoio diagnóstico e tratamentos pós-exposição nos serviços de saúde, pois muitas pessoas, e entre elas estão os jovens, podem estar vivendo com o vírus sem terem conhecimento.

A razão de sexos vem diminuindo, conforme demonstrado por estudo realizado no Estado do Ceará22 ${ }^{22}$ portanto, para conter a feminilização do HIV/aids deveriam ser considerados para adoção de medidas profiláticas, as condições socioeconômicas, culturais e relações de poder entre homens e mulheres de cada região país ${ }^{1}$.

\section{CONCLUSÃO}

A principal limitação deste estudo foi o uso de um banco de dados fechado que não permitiu aprofundar discussões acerca dos processos que determinam o agravo HIV/aids e sobre categorias relevantes para a análise no campo da Saúde Coletiva, como gênero e classe social dos indivíduos notificados.

Conclui-se que, a partir dos dados analisados, foi possível compreender que nos últimos anos a epidemia de HIV/aids vem aumentando em adultos jovens e que o perfil da epidemia em mulheres jovens $2^{2}$ RS do Paraná é convergente com as características da epidemia nacional. Há concentração de casos de mulheres que viviam com HIV/aids em grandes centros urbanos, tendo como provável modo de transmissão a relação heterossexual. As mais atingidas foram mulheres que viviam 
em condições de vida precárias, com baixa escolaridade, e que exerciam a função de dona de casa ou estavam desempregadas.

Ações que enfoquem a prevenção e o diagnóstico precoce para mulheres fora do ciclo gravídico-puerperal são urgentes, pois muitas mulheres jovens podem estar vivendo com HIV e/ou aids sem sorologia anti-HIV conhecida.

Além disso, é preciso investir em políticas públicas, pois o problema HIV/aids em mulheres jovens extrapola o limite do biológico, inclui processos determinados pela escolaridade, renda, ocupação e gênero. Portanto, o fenômeno não exige apenas intervenções assistenciais e medicamentosas, mas, principalmente, ações que fortaleçam os vínculos entre os serviços de saúde e os indivíduos que estão nos territórios.

\section{AGRADECIMENTOS}

À Coordenação de Aperfeiçoamento de Pessoal de Nível Superior, pela Bolsa Social concedida a uma das autoras deste manuscrito.

\section{REFERÊNCIAS}

1.Campany LNS, Amaral DM, Santos RNOL. HIV/aids no Brasil: feminização da epidemia em análise. Rev Bioet. 2021;29(2):374-83. doi: https://doi.org/10.1590/1983-80422021292475

2. United Nations Programme on HIV/aids (UNAIDS) [Internet]. Estatísticas globais sobre HIV; c2021 [citado em 11 out. 2021]. Disponível em: https://unaids.org.br/estatisticas

3. Ministério da Saúde(BR). Bol Epidemiol HIV-Aids: 2020. Brasília: Min Saúde; 2020. 68p. Disponível em: http://www.aids.gov.br/pt-br/pub/2020/boletim-epidemiologicohivaids-2020

4. Schaurich D, Coelho DF, Motta MGC. A cronicidade no processo saúde-doença: repensando a epidemia da AIDS após os antirretrovirais. Rev Enferm UERJ. 2006;14(3):455-62. Disponível em: https://lume.ufrgs.br/handle/10183/107165

5. Nakamura E, Egry EY, Campos CMS, Nichiata LYI, Chiesa AM, Takahashi RF. O potencial de um instrumento para o reconhecimento de vulnerabilidades sociais e necessidades em saúde: saberes e práticas em saúde coletiva. Rev Esc Enferm. USP. 2009;17(2): 117-123. doi: https://doi.org/10.1590/S010411692009000200018 
6. Ministério da Saúde(BR). HIV em Gestantes, Aids e Mortalidade por Aids. Bol Epidemiol HIV/AIDS. 2015;4(1):4-47. Disponível em: http://www.aids.gov.br/ptbr/pub/2015/boletim-epidemiologico-hivaids-2015

7. Secretaria de Estado da Saúde (PR). Plano Estadual de Saúde do Paraná 20162019. Curitiba: Secretaria de Estado da Saúde; 2016. Disponível em: https://www.conass.org.br/pdf/planos-estaduais-desaude/PR PlanoEstadualSaude2016MioloAlt.pdf

8. Breilh J. Epidemiologia crítica: ciência emancipadora e interculturalidade. Rio de Janeiro: Fundação Oswaldo Cruz; 2006.

9. Paim JS, Almeida Filho N. Saúde coletiva: teoria e prática. Rio de Janeiro: MedBook; 2014.

10. Almeida Filho N, Barreto ML. Epidemiologia e Saúde: fundamentos, métodos e aplicações. Rio de Janeiro: Guanabara Koogan; 2013.

11. Ministério do Trabalho e Emprego (BR). Classificação Brasileira de Ocupações. 3ed. Brasília: Ministério do Trabalho e Emprego; 2010. Disponível em: https://wp.ufpel.edu.br/observatoriosocial/files/2014/09/CBO-Livro-1.pdf

12. Ministério da Saúde (BR). Resolução n. 466, de 12 de dezembro de 2012. [Internet]. c2012. [citado em 17 Fev. 2019]. Disponível em: http://bvsms.saude.gov.br/bvs/saudelegis/cns/2013/res0466 1212 2012.html

13. Ministério da Saúde (BR). HIV em gestantes. Bol Epidemiol HIV/AIDS. 2016;5(1):3-53. Disponível

em: http://www.aids.gov.br/system/tdf/pub/2016/59427/boletim 20161 pdf 16375.p df file $=1 \&$ type $=$ node $\&$ id $=59427 \&$ force $=1$

14. São Paulo. Secretaria de Estado da Saúde. HIV-AIDS. Bol Epidemiol Aids/DST. 2016;33(1):4-104. Disponível em: http://www.saude.sp.gov.br/resources/crt/vig.epidemiologica/boletimepidemiologico-crt/boletim epidemiologico 2016.pdf?attach=true

15. Instituto Paranaense de Desenvolvimento Econômico e Social (IPARDES). [Internet]. Cadernos Municipais. c2017 [Acesso em 16 Jul. 2019]. Disponível em: http://www.ipardes.pr.gov.br/Pagina/Cadernos-municipais

16. Galvão JMV, Costa ACM, Galvão JV. Demographic and socio-demographic profile of people living with HIV/AIDS. Rev Enferm UFPI. 2017;6(1):4-8. Disponível em: https://revistas.ufpi.br/index.php/reufpi/article/view/5533/0

17. Ministério da Saúde (BR). Portaria MS n 3.947 de 25 novembro de 1998. Brasília: Ministério da Saúde; 1999. Seção 1. Disponivel em: http://portalsinan.saude.gov.br/images/documentos/Legislacoes/Portaria 3947 a tributos comuns.pdf

18. Secretaria Municipal de Saúde de Curitiba (PR). Boletim Epidemiológico HIV/aids. Curitiba: Secretaria Municipal de Saúde; 2016. p. 52. 
19. Gonçalves H, Machado EC, Soares ALG, Camargo-Figuera FA, Seerig LM, Mesenburg MA, et al. Início da vida sexual entre adolescentes (10 a 14 anos) e comportamentos em saúde. Rev Bras Epidemiol. 2015;18(1):1-18. doi: 10.1590/1980-5497201500010003

20. Acosta LMW, Gonçalves TR, Barcellos NT. Coinfecção HIV/sífilis na gestação e transmissão vertical do HIV: um estudo a partir de dados da vigilância epidemiológica. Rev Panam Salud Publica. 2016;40(6):435-42.Disponível em: https://www.scielosp.org/pdf/rpsp/2016.v40n6/435-442

21. Ministério da Saúde (BR). Gestação de alto risco: manual técnico. Brasília: Ministério da Saúde; 2012. Disponível em: https://bvsms.saude.gov.br/bvs/publicacoes/manual tecnico gestacao alto risc o.pdf

22. Pedrosa NL, Paiva SS, Almeida RLF, Holanda ER, Kerr LRFS, Galvão MTG. Série histórica da AIDS no Estado do Ceará, Brasil. Ciênc Saúde Coletiva. 2015;20(4):1177-84. doi: https://doi.org/10.1590/1413-81232015204.00582014 